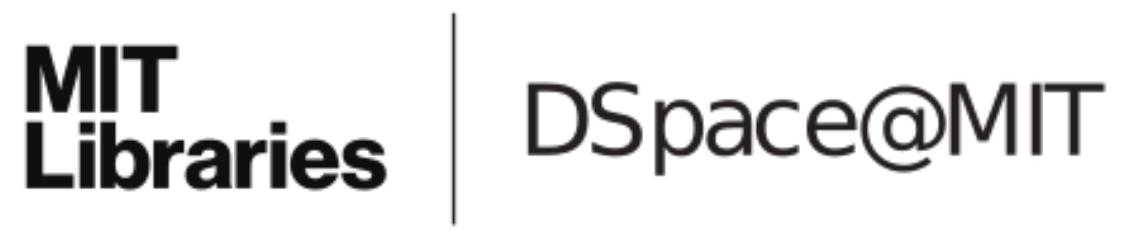

\author{
MIT Open Access Articles
}

Experiment Turbulence Compensation of 50Gbaud/s Orbital-Angular-Momentum QPSK Signals using Intensity-only based SPGD Algorithm

The MIT Faculty has made this article openly available. Please share how this access benefits you. Your story matters.

Citation: Xie, Guodong, Yongxiong Ren, Hao Huang, Martin P. Lavery, Nisar Ahmed, Yan Yan, Changjing Bao, et al. "Experiment Turbulence Compensation of 50-Gbaud/s OrbitalAngular-Momentum QPSK Signals Using Intensity-Only Based SPGD Algorithm." Optical Fiber Communication Conference (2014).

As Published: http://dx.doi.org/10.1364/OFC.2014.W1H.1

Publisher: Optical Society of America

Persistent URL: http://hdl.handle.net/1721.1/93907

Version: Author's final manuscript: final author's manuscript post peer review, without publisher's formatting or copy editing

Terms of use: Creative Commons Attribution-Noncommercial-Share Alike 


\title{
Experiment Turbulence Compensation of 50-Gbaud/s Orbital-Angular-Momentum QPSK Signals Using Intensity-only based SPGD Algorithm
}

\author{
Guodong Xie $^{1 *}$, Yongxiong Ren ${ }^{1}$, Hao Huang ${ }^{1}$, Martin P. J. Lavery ${ }^{2}$, Nisar Ahmed ${ }^{1}$, Yan Yan ${ }^{1}$, Changjing Bao ${ }^{1}$, \\ Long $\mathrm{Li}^{1}$, Zhe Zhao ${ }^{1}$, Yinwen Cao ${ }^{1}$, Moshe Willner ${ }^{1}$, Miles J. Padgett ${ }^{2}$, Moshe Tur ${ }^{3}$, Samuel J. Dolinar ${ }^{4}$, \\ Robert W. Boyd ${ }^{5}$, Jeffrey H. Shapiro ${ }^{6}$, and Alan E. Willner ${ }^{1}$ \\ 1. Dept. of Electrical Engineering, University of Southern California, Los Angeles, CA 90089, USA, Email: guodongx@usc.edu \\ 2. School of Physics and Astronomy, University of Glasgow, Glasgow, G12 8QQ, UK \\ 3. School of Electrical Engineering, Tel Aviv University, Ramat Aviv 69978, ISRAEL \\ 4. Jet Propulsion Laboratory, California Institute of Technology, Pasadena, CA 91109, USA \\ 5. Dept. of Physics and Astronomy, The Institute of Optics, University of Rochester, Rochester, NY 14627, USA \\ 6. Research Laboratory of Electronics, Massachusetts Institute of Technology, Cambridge, MA 02139, USA
}

\begin{abstract}
An intensity-based algorithm is employed to derive the correction phase pattern for a distorted probe orbital-angular-momentum (OAM) beam. This correction pattern is used to compensate the distortions of three multiplexed OAM beams, each carrying a 50-Gbaud QPSK signal. The crosstalk between channels is reduced by $>5 \mathrm{~dB}$ with this approach.

OCIS codes: (010.1330) Atmospheric turbulence, (010.1285) Atmospheric correction
\end{abstract}

\section{Introduction}

Orbital-angular-momentum (OAM) has emerged as an optical communication technique for simultaneously transmitting spatially overlapping orthogonal modes that can be efficiently multiplexed at the transmitter and demultiplexed at the receiver. An OAM beam has a helical phase front that twists during propagation, with the number of $2 \pi$ phase shifts across the beam front corresponding to the OAM mode order or charge [1]. Studies have shown the successful transmission of multiple OAM modes both in free-space and via a special optical fiber, achieving capacities in excess of $1 \mathrm{Tbit} / \mathrm{s}[2,3]$.

Atmospheric turbulence presents a critical limitation on free-space optical (FSO) communication that uses a single non-OAM spatial mode. It degrades the Gaussian beam because of the beam spread, angle-of-arrival spread, and scintillation it creates [4]. Partial compensation for these effects can be achieved by monitoring the received beam and using feedback to control an adaptive optical element that undoes the turbulence-induced phase fluctuations in the receiver's entrance pupil [4].

The scenario with an OAM-based FSO system is far more challenging than single-mode operation because turbulence causes crosstalk between the modes [5-7]. Recently, turbulence compensation has been demonstrated in an FSO OAM system [8]. A Gaussian beam of the same wavelength but orthogonally polarized to the OAM beams was used to probe the turbulence, and a wave-front sensor was used to measure the phase-front distortion due to the turbulence. This receiver-pupil phase distortion was then removed with a spatial light modulator (SLM) prior to detection [8]. However, this method requires direct measurements of phase-front distortion by a relatively complicated Shack-Harman wave-front sensor, which can only retrieve the phase-front of a light beam without vortices (e.g., the probe beam needs to be a non-OAM beam). A laudable goal would be to replace the wave-front sensor approach with a simpler method in which only intensity measurements by an intensity-only camera are required to derive the correction phase pattern. In this case, the probe beam which could also carry data information can be either an OAM or non-OAM beam.

In this paper, we use the stochastic-parallel-gradient-descent (SPGD) algorithm [9,10] to derive the phase correction pattern based on the measured intensity profile of the distorted probe OAM beam. A single OAM beam is sent though the emulated turbulence to probe the wave-front distortion and is used to derive the phase correction pattern. This pattern is used to simultaneously compensate for multiple multiplexed OAM beams under the same turbulence condition. Experimental results indicate that the crosstalk of each OAM channel can be reduced by $>5 \mathrm{~dB}$ using this technique.

\section{Concept and principle}

Figure 1(a) shows the concept of correction pattern derivation using intensity-only SPGD algorithm. The phasefront and intensity profile of an OAM beam will be distorted as it propagates through the turbulent media. In order to correct this distortion, the intensity profile of the probe beam is recorded and then processed by the SPGD algorithm $[9,10]$ to produce a feed-back correction phase mask at the receiver entrance pupil. As indicated in [11], mode purity of an OAM beam monotonically increases with the increment of the intensity correlation. Here the intensity correlation is defined as the correlation coefficient between the far-field intensity profile of the received probe OAM beam and its ideal intensity distribution. This suggests that the intensity profile can be used to derive the error signal in the feed-back loop to update the correction phase pattern. Starting with an initial phase correction 
pattern, the SPGD algorithm takes the intensity correlation of the probe beam as the input, and it outputs the updated phase correction pattern. Fig. 1(b) shows the block diagram of the SPGD algorithm as described in $[9,10,12]$.

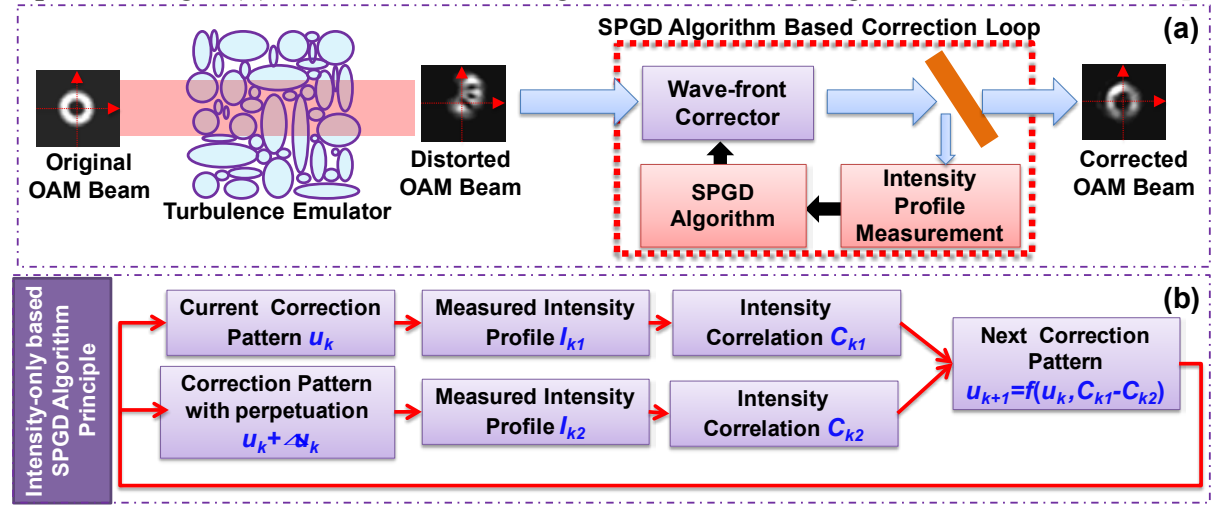

Figure 1 (a) Concept of the distortion compensation of an OAM beam. (b) Block diagram of SPGD algorithm. Intensity correlation is the correlation coefficient between the measured far-field intensity profile of the received probe beam and its ideal intensity distribution.

\section{Experimental setup and results}

Figure 2 shows the experimental setup of phase correction pattern derivation using SPGD algorithm. The generated 50 -Gbaud QPSK signal at $1550 \mathrm{~nm}$ is split into 3 copies, each of which is de-correlated with one another. The three fiber branches are fed into the collimators, which emit collimated Gaussian beams. One beam (branch (1)) is converted to OAM +3 by SLM-1, while the others (branches (2) and (3) are converted to OAM +1 and OAM +5 by SLM-2 and SLM-3, respectively. Branches (2) and (3) are turned on only during the BER measurement. The OAM beam(s) propagate(s) through a turbulence emulator, which consists of a rotatable stage and a phase screen plate, to simulate the atmospheric effects $[13,14]$. The phase screen plate with a Fried's parameter of $r_{0}=1 \mathrm{~mm}$ at $1550 \mathrm{~nm}$ follows Kolmogorov spectrum statistics, which is mounted into the rotatable stage to emulate a $1 \mathrm{~km}$ turbulence channel. An infrared (IR) camera captures the far-field intensity profile of the beam. The intensity correlation between this profile and the beam's ideal intensity is used as the input of the SPGD algorithm. The SPGD algorithm could generate an optimal correction pattern that maximizes this intensity correlation. The correction pattern is then loaded onto SLM-4 to compensate the turbulence-induced wave-front distortions. For the bit error rate (BER) measurement, the $\mathrm{OAM}+3$ is down-converted into to a Gaussian beam by loading an inverse spiral phase pattern of $\mathrm{OAM}+3$ onto SLM-5. This Gaussian beam is then coupled into a single mode fiber and sent for coherent detection and off-line digital signal processing.

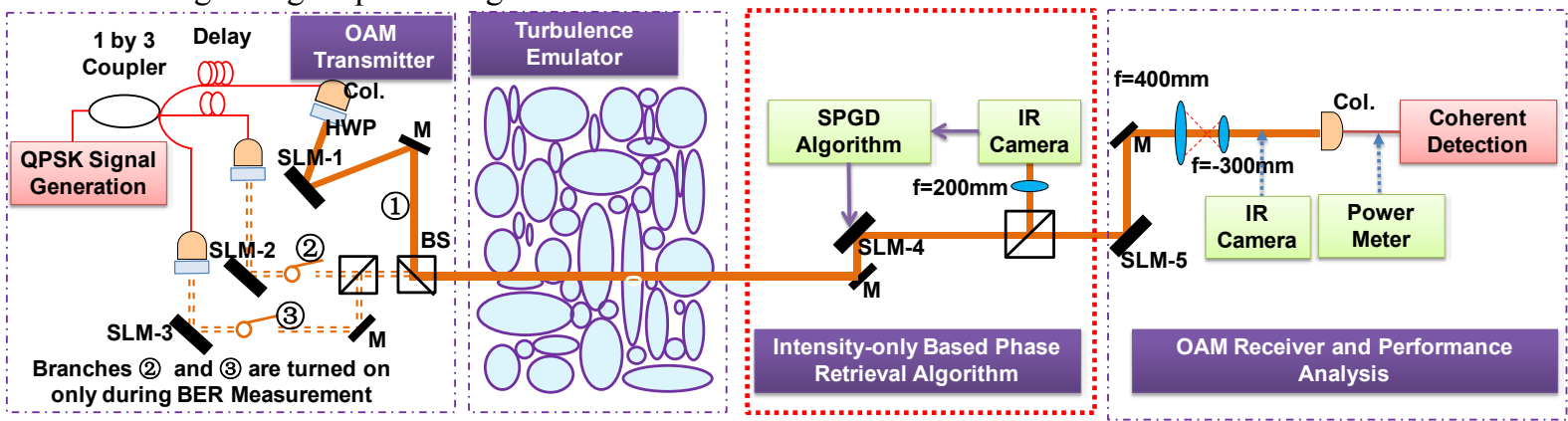

Figure 2 Experimental setup. Col.: collimator; HWP: half wave plate; SLM: spatial light modulator; M: mirror; BS: beam splitter; IR: infrared. The correction pattern is generated when branches (2) and (3) are turned off; and this correction pattern is used to compensate for the system when branches (2) and (3) are turned on and $\mathrm{OAM}+1,+3$ and +5 are transmitted simultaneously.

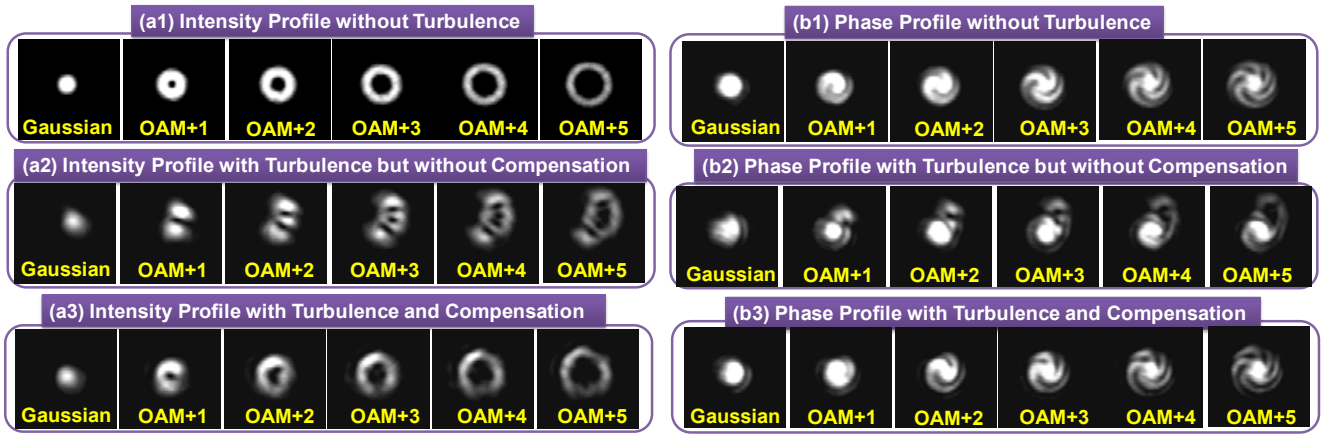

Figure 3 Far-field intensity profiles and phase interference patterns for different OAM beams with and without compensation under a specific turbulence realization. The phase interference patterns are generated by using a Gaussian beam to interfere with the uncorrected/corrected beams. The atmospheric coherence length of the turbulence is $1 \mathrm{~mm}$. Correction pattern is calculated by the SPGD algorithm when only OAM +3 is transmitted and this pattern is employed to compensate Gaussian beam and OAM +1 to +5 . 
Figure 3 shows the far-field intensity profiles and phase interference patterns for different OAM beams with and without compensation for a static turbulence realization. As OAM beams have different beam sizes, they experience different turbulence strengths, which is characterized by $D / r_{0}$, with $D$ the diameter of the beam size at the incident plane of the turbulence emulator plane. Here, $D / r_{0}$ for $\mathrm{OAM}+1$ to $\mathrm{OAM}+5$ are $1.5,1.8,2.1,2.4$ and 2.7 respectively. The correction pattern is calculated by the SPGD algorithm when only OAM +3 is transmitted. Subsequently this correction pattern is used to correct the wave-front distortion of the other beams. One can see from both the intensity profiles and the phase interference patterns that the distorted OAM beams are improved. The intensity correlations for Gaussian beams and $\mathrm{OAM}+1$ to +5 are increased from $0.40,0.59,0.60,0.60,0.56$, and 0.52 (Fig. 3(a2)) to 0.81, $0.91,0.94,0.94,0.93$, and 0.90 (Fig. 3(a3)), respectively.

Figure 4(a) shows the intensity correlations of the probe beam $(\mathrm{OAM}+3)$ with and without compensation for different realizations (i.e., repeat the measurements for 9 times) under the same static turbulence strength $\left(D / r_{0}=\right.$ 2.1, $\tilde{C}_{n}^{2}=3.6 \times 10^{-15} \mathrm{~m}^{-2 / 3}$ where $\tilde{C}_{n}^{2}$ is the atmospheric structure constant). Measured results indicate that the intensity correlation can be improved to be above 0.9. Figure 4(b) shows intensity correlation as a function of iteration number (i.e., the number of repetitions that the algorithm executed) of the algorithm. One can observe that the algorithm could converge after $\sim 50$ iterations. Figure $4(\mathrm{c})$ shows the crosstalk from OAM +3 to its neighboring modes. Without compensation, most power of $\mathrm{OAM}+3$ spreads to its neighboring modes $(\mathrm{OAM}+1$ to +5$)$. While with compensation, the power of $\mathrm{OAM}+3$ is increased and the power leaked to its neighbors decreases simultaneously.

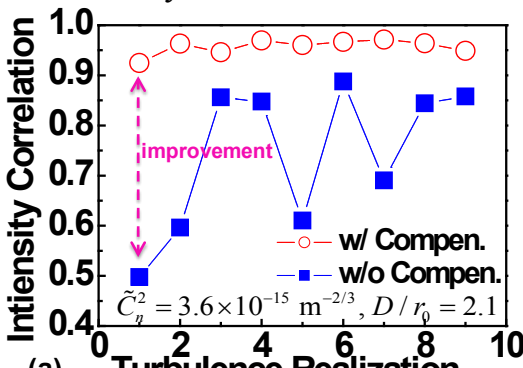

(a)

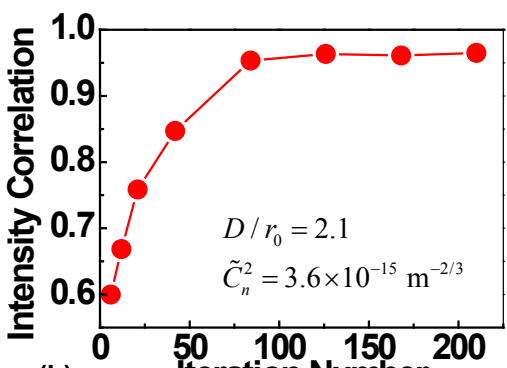

(b)

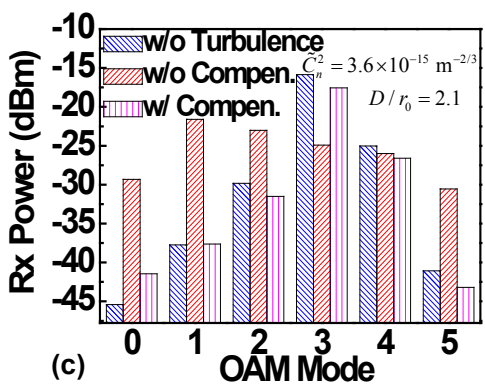

Figure 4 (a) Intensity correlation of OAM +3 with and without compensation when only branch (1) is turned on. Here we took 9 measurements in sequence (i.e., 9 turbulence realizations) under the same turbulence condition, which has the turbulence strength of $D / r_{0}=2.1$. (b) Intensity correlation as a function of the iteration number (i.e., the number of repetitions that the algorithm executed). (c) The received power of different OAM modes before and after compensation when only OAM +3 is transmitted.

The phase correction pattern derived from the probe beam can be used to compensate multiple spatially multiplexed OAM beams under the same turbulence strength. Following above, here we demonstrated simultaneous compensation of three channels $(\mathrm{OAM}+1, \mathrm{OAM}+3$ and $\mathrm{OAM}+5)$. Figure 5(a) shows BERs of one of the three channels $(\mathrm{OAM}+3)$ before and after compensation. The BER can barely reach the forward error correction (FEC) limit of 3.8e-3 without compensation, while after the compensation, BER can be reduced to $<1 \mathrm{e}-4$. The measured crosstalk from $\mathrm{OAM}+1$ and +5 channels to $\mathrm{OAM}+3$ channel is reduced from $-7.03 \mathrm{~dB}$ to $-17 \mathrm{~dB}$ by compensation. Figure 5(b) shows another realization under the same turbulence condition. The power crosstalk from OAM +1 and +5 channels to $\mathrm{OAM}+3$ channel is mitigated from $-10.5 \mathrm{~dB}$ to $-15.7 \mathrm{~dB}$ by compensation and the power penalty is improved by $\sim 4 \mathrm{~dB}$ at FEC limit.
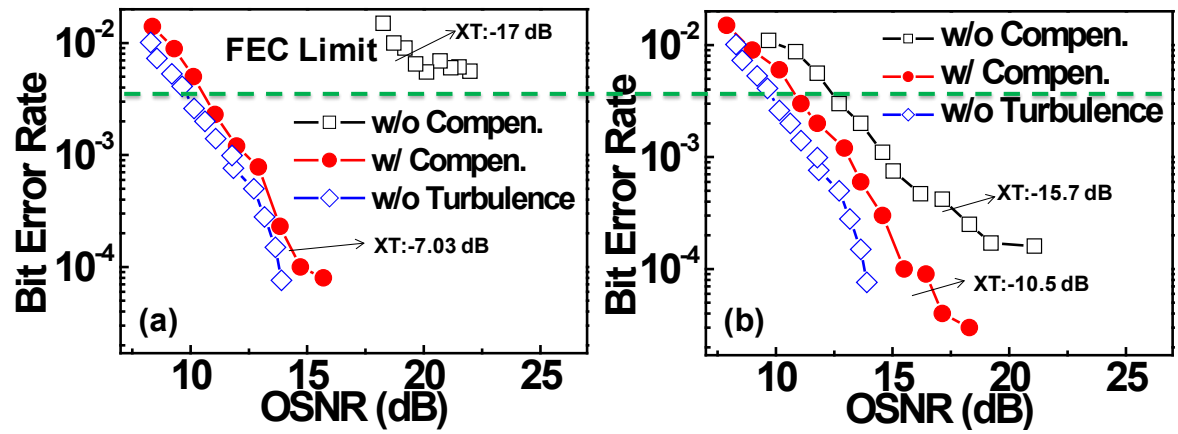

Figure 5 Measured BER curves when simultaneously compensating multiplexed $\mathrm{OAM}+1, \mathrm{OAM}+3$ and $\mathrm{OAM}+5$ using the correction phase pattern derived from the probe beam. (a) and (b) are two different realizations under the same static turbulence strength. (a) Measured crosstalk from $\mathrm{OAM}+1$ and +5 channels to $\mathrm{OAM}+3$ channel is $-7.03 \mathrm{~dB}$ without compensation and $-17 \mathrm{~dB}$ with compensation. (b) Measured crosstalk is $10.5 \mathrm{~dB}$ without compensation and $-15.7 \mathrm{~dB}$ with compensation. XT:crosstalk.

Acknowledgement We acknowledge supports of DARPA under the InPho program and Intel Labs University Research Office.

\section{References}

[1] G. Gibson et al., OE 12, 5448(2004)

[2] J. Wang et al., Nat. Photon. 6, 488 (2012)

[3] B. Nenad et al., Science 340, 1545 (2013)

[4] L. Andrews et al., SPIE Press, 2005

[5] M. Malik et al., OE 20, 13159 (2012)
[6] B. Rodenburg et al., OL 37, 3735 (2012)

[7] J. Anguita et al., AO 47, 2414 (2008)

[8] Y. Ren et al., ECOC2013, We.3.D.1

[9] H. Ma et al., OL 35, 2973 (2010)

[10] W. Thomas et al., SPIE2003, 5162
[11] H. Huang et al., OL 38, 2348 (2013)

[12] R. Noll et al., JOSA 66, 207 (1976)

[13] Y. Ren et al., OL 38, 4062 (2013)

[14] N. Chandrasekaran et al., SPIE2012, 851808 